\title{
Kinect v2 for Mobile Robot Navigation: Evaluation and Modeling
}

\section{Conference Paper}

Author(s):

Fankhauser, Péter; Bloesch, Michael; Rodriguez, Diego; Kaestner, Ralf; Hutter, Marco (1); Siegwart, Roland

Publication date:

2015-09-10

Permanent link:

https://doi.org/10.3929/ethz-a-010513824

Rights / license:

In Copyright - Non-Commercial Use Permitted

Originally published in:

https://doi.org/10.1109/ICAR.2015.7251485 


\title{
Kinect v2 for Mobile Robot Navigation: Evaluation and Modeling
}

\author{
Péter Fankhauser*, Michael Bloesch*, Diego Rodriguez*†, \\ Ralf Kaestner*, Marco Hutter*, Roland Siegwart* \\ *Autonomous Systems Lab, ETH Zurich, Switzerland \\ ${ }^{\dagger}$ Robotics and Embedded Systems, Technische Universität München, Germany
}

\begin{abstract}
With the introduction of the Microsoft Kinect for Windows v2 (Kinect v2), an exciting new sensor is available to robotics and computer vision researchers. Similar to the original Kinect, the sensor is capable of acquiring accurate depth images at high rates. This is useful for robot navigation as dense and robust maps of the environment can be created. Opposed to the original Kinect working with the structured light technology, the Kinect $\mathrm{v} 2$ is based on the time-of-flight measurement principle and might also be used outdoors in sunlight. In this paper, we evaluate the application of the Kinect $v 2$ depth sensor for mobile robot navigation. The results of calibrating the intrinsic camera parameters are presented and the minimal range of the depth sensor is examined. We analyze the data quality of the measurements for indoors and outdoors in overcast and direct sunlight situations. To this end, we introduce empirically derived noise models for the Kinect $\mathrm{v} 2$ sensor in both axial and lateral directions. The noise models take the measurement distance, the angle of the observed surface, and the sunlight incidence angle into account. These models can be used in post-processing to filter the Kinect $v 2$ depth images for a variety of applications.
\end{abstract}

\section{INTRODUCTION}

Mobile robot navigation requires a robot to perceive its surroundings while moving through previously unseen environments. Range sensors provide the means to spatially capture the terrain and objects around the robot. Several requirements such as size, weight, resolution, update rate, field of view, and robustness against external light conditions influence the selection of the sensory setup. With the introduction of the original Microsoft Kinect, a low-cost depth sensor for fast and highquality dense depth images was made available. Its release had a big impact in robotics and we have seen a multitude of applications (see [1] for an overview). However, the underlying structured-light technology is unsuitable in sunlight conditions [1] which limits its range to indoor applications. The second generation of the device, the Microsoft Kinect for Windows v2 (Kinect v2) [2], was introduced in 2013 and has been available to researchers since July 2014. The new version of the Kinect is based on the time-of-flight (ToF) principle and offers a higher resolution and a wider field of view in comparison to its predecessor. Depending on the intensity of the signal emitted to determine the ToF, the sensor might be applicable outdoors. In this paper, we examine the potential of the Kinect v2 for robot navigation as shown in Fig. 1 as example application. We evaluate the systematic and non-systematic errors, determine the short-range measurement capability, and characterize the influence of the ambient light for indoor, overcast, and direct sunlight situations. To other researchers and developers, we

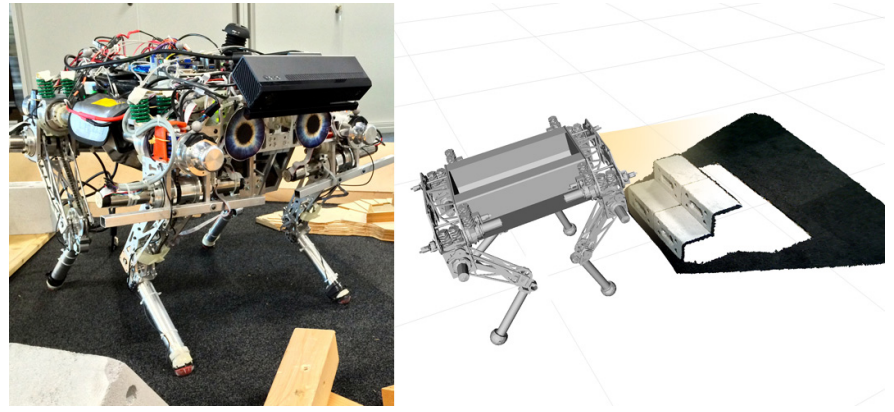

Fig. 1. The quadruped robot StarlETH [3] uses a Kinect v2 sensor for rough terrain mapping and locomotion [4].

provide our adaption of the available open-source driver for the Kinect v2 with interface to the Robot Operating System (ROS) as easy-to-install, package-based distribution for Linux 1

Because of its recent release, only little work has been published on the Kinect v2. The works of [5, 6, 7] compare the performance of the original Kinect with the Kinect v2 and conclude that the Kinect v2 shows better performance in terms of systematic and non-systematic errors for depth measurements. In [5, 8, 9] it has also been exemplarily shown that the Kinect v2 is able to capture data for shadow and direct sunlight situations. Other analyzed aspects include the influence of surface color and roughness on the depth measurements [5, 6, 9], pre-heating of the device [6, 9], calibration [8, 9], and measurements through water [8]. Our work is motivated to provide a model of the non-systematic errors of the Kinect v2 depth camera for further processing of the measurements such as in [4]. To this end, the contribution of this paper is the modeling of the noise characteristics of the Kinect v2 depth sensor in both axial and lateral direction. We empirically derive our noise model as a function of the measurement distance and the angle of the observed surface. While we can characterize indoor and overcast situations with the same approach, we extend our model by the incidence angle for direct sunlight. Additionally, an analysis of the minimal measurement range for different surfaces is presented.

The remainder of this paper is structured as follows. In Section $\mathrm{II}$ we give an overview on the technology behind the Kinect v2 and the key specifications of the product. Section III analyzes the results of a standard calibration procedure for the intrinsic parameters of its infrared (IR) camera. Sections IV and $\mathrm{V}$ present our experimental setup and the resulting

\footnotetext{
${ }^{1}$ The libfreenect 2 package can be installed from ppa:ethz-asl/drivers

${ }^{2}$ The ros-indigo-kinect 2 package can be installed from ppa:ethz-as1/ros
} 
TABLE I. KINECT V2 SPECIFICATIONS

\begin{tabular}{|c|c|c|c|}
\hline & & $\begin{array}{l}\text { PrimeSense } \\
\text { Carmine 1.08 }\end{array}$ & Kinect v2 \\
\hline \multirow{4}{*}{$\begin{array}{l}\text { Infrared/depth } \\
\text { camera }\end{array}$} & Resolution & $320 \times 240 \mathrm{px}$ & $512 \times 424 \mathrm{px}$ \\
\hline & Field of view $(\mathrm{h} \times \mathrm{v})$ & $57.5^{\circ} \times 45.0^{\circ}$ & $70.6^{\circ} \times 60.0^{\circ}$ \\
\hline & Angular resolution & $0.18^{\circ} / \mathrm{px}$ & $0.14^{\circ} / \mathrm{px}$ \\
\hline & Operating range & $0.8-3.5 \mathrm{~m}$ & $0.5-4.5 \mathrm{~m}$ \\
\hline \multirow{2}{*}{ Color camera } & Resolution & $640 \times 480 \mathrm{px}$ & $1920 \times 1080 \mathrm{px}$ \\
\hline & Field of view $(h \times v)$ & $57.5^{\circ} \times 45.0^{\circ}$ & $84.1^{\circ} \times 53.8^{\circ}$ \\
\hline \multicolumn{2}{|l|}{ Frame rate } & $30 \mathrm{~Hz}$ & $30 \mathrm{~Hz}$ \\
\hline \multicolumn{2}{|c|}{ Minimum latency } & & $20 \mathrm{~ms}$ \\
\hline \multicolumn{2}{|c|}{ Shutter type } & & Global shutter \\
\hline \multicolumn{2}{|c|}{ Dimensions $(\mathrm{w} \times \mathrm{d} \times \mathrm{h})[\mathrm{mm}]$} & $180 \times 35 \times 25$ & $249 \times 66 \times 67$ \\
\hline \multicolumn{2}{|c|}{ Mass (without cables) } & $160 \mathrm{~g}$ & $970 \mathrm{~g}$ \\
\hline \multicolumn{2}{|c|}{ Connection type } & USB 2.0 & USB 3.0 \\
\hline \multicolumn{2}{|l|}{ Voltage } & $5 \mathrm{~V} \mathrm{DC}$ & $12 \mathrm{~V} \mathrm{DC}$ \\
\hline \multicolumn{2}{|l|}{ Power usage } & $2.25 \mathrm{~W}$ & $\sim 15 \mathrm{~W}$ \\
\hline \multicolumn{2}{|l|}{$\overline{\text { Price }}$} & 200 USD & 200 USD \\
\hline
\end{tabular}

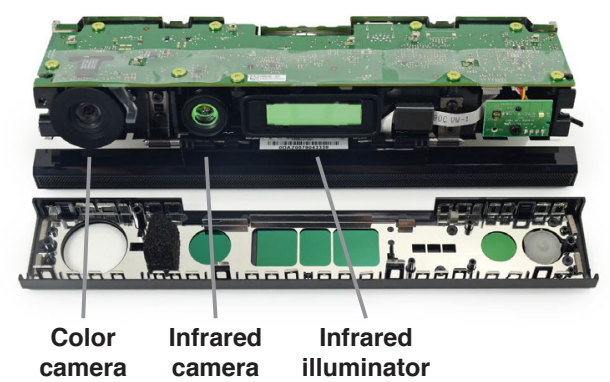

Fig. 2. The Kinect v2 senses depth by measuring the time it takes for light to travel from the infrared illuminator to the object and back to the infrared camera. Image taken from [12].

noise model for indoor and outdoor (overcast and direct sunlight) conditions. Finally, a conclusion of the results and our experience is drawn in Section $\overline{\mathrm{VI}}$

\section{THE KINECT V2 SENSOR}

We summarize a list of key specifications of the Kinect v2 sensor in Table II The device is similar in size compared to the original Kinect for Xbox 360, but bulkier and heavier in relation to the PrimeSense Carmine and Asus Xtion series, and other ToF-sensors [10]. A considerable difference between the Kinect v2 and existing ToF-sensors is the resolution of the depth camera. Whereas other ToF-sensors have a resolution of typically up to $200 \times 200 \mathrm{px}(320 \times 240 \mathrm{px}$ for the SoftKinetic DS325) [6], the Kinect v2 features a higher resolution of $512 \times 424 \mathrm{px}$.

The Kinect v2 depth sensor is based on the time-of-flight measurement principle. A strobed infrared light (see Fig. 2) illuminates the scene, the light is reflected by obstacles, and the time of flight for each pixel is registered by the infrared camera. Internally, wave modulation and phase detection is used to estimate the distance to obstacles (indirect ToF). Details on the depth measurement method of the Kinect v2 are given in [11].

Integrating the sensor on a robotic system requires knowledge of the coordinate system associated with the depth measurements. Fig. 3 illustrates the position of the IR image sensor that we have acquired by manual measurements and the calibration

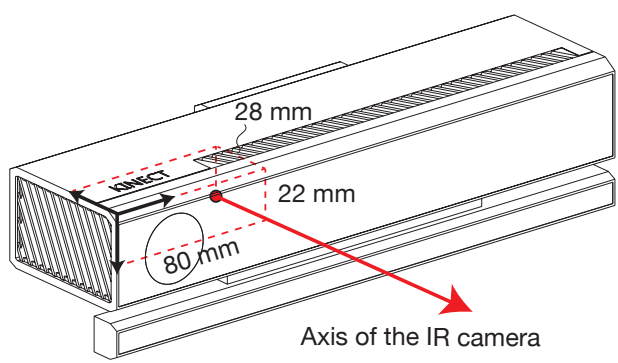

Fig. 3. Estimated optical center of the infrared camera. The axis of the IR camera is assumed to follow perpendicularly through the front plate.

procedure described in Section III We assume that the IR camera axis is perpendicular to the front plate of the Kinect v2.

In the course of this work, we have used the open-source Kinect v2 driver by Blake et al. [13] and its associated ROS packages contributed by Wiedemeyer [14]. For simple installation, we contributed to these projects by providing packagebased deployment of libfreenect 2 and its development headers for Ubuntu 14.04 LTS (Trusty Tahr) ${ }^{3}$ The integration supports OpenGL and OpenCL accelerated depth package processing in addition to the standard CPU-based method. Analogously, a project for Kinect v2 ROS integration from binary packages has been initiated $4^{4}$

\section{Systematic ERRORs \& CALIBRATION}

Depth measurements from ToF cameras are generally corrupted by systematic, reproducible errors and non-systematic, random errors. In this section, we analyze the systematic errors that remain after a calibration procedure. Different types of systematic errors have been studied [10], and we set the focus on the depth distortion and the amplitude-related errors. We neglect integration-time-related errors, as the integration time (IT) cannot be selected by the user for the Kinect v2. Before taking measurements, the Kinect v2 was run for more than 20 minutes to account for temperature-related errors of the retrieved data [6]. We calibrated the intrinsic parameters of the IR camera with the help of a checkerboard and the software from Wiedemeyer [14] which uses the OpenCV calibration. The average reprojection error after calibration was below $0.5 \mathrm{px}$.

We estimated the depth distortion as the difference between the mean measured depth and the real distance. For this, the depth sensor was positioned to perpendicularly face a white wall at several distances. Depth measurements were taken from a small patch at the image center and averaged over 100 samples. The real distance was measured with a tape measure with an accuracy of $\pm 1 \mathrm{~mm}$. Fig. 4 shows the resulting distance offsets for ranges between 0.7 an $2.75 \mathrm{~m}$. The mean of the offset distances was used to determine the position of the coordinate system associated with the depth measurements along the camera axis (Fig. 3). The measured depth distortion was found to oscillate between $\pm 6 \mathrm{~mm}$, which is often referred to as wiggling error of ToF cameras [10], [15].

Amplitude-related errors typically occur due to inconsistence of the infrared light reflected from objects. This can be

\footnotetext{
${ }^{3}$ Available from https://github.com/ethz-asl/libfreenect2/tree/remake

${ }^{4}$ Available from https://github.com/ethz-asl/kinect2-ros
} 


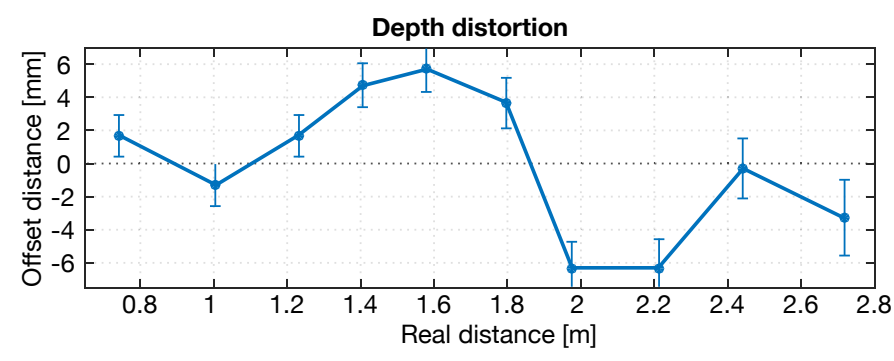

Fig. 4. The offset distance describes the error between the averaged depth measurement and the true distance. The whiskers depict one standard deviation $\sigma_{z}$ above and below the mean of the data. The depth distortion follows an oscillatory pattern over the range of distances. This wiggling effect appears due to irregularities in the modulation process of the illuminator [10], [15].

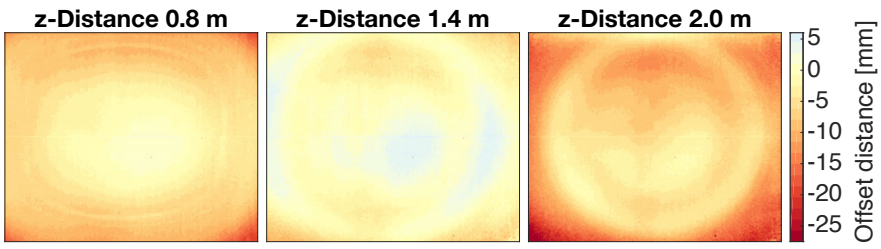

Fig. 5. Colored depth errors between an averaged snapshot of a flat wall and a reference plane (the wall angle was obtained through plane fitting). Depth overestimation can be observed due to low illumination at the image borders.

caused by non-uniform illumination and scenes with objects at different distances and surfaces with different reflectivities. We analyzed the amplitude-related errors by comparing timeaveraged data from the sensor facing a white wall with data of a plane fit. The data was averaged over 100 depth images of the same scene, and a plane was fit using a linear least squares regression. Fig. 5 shows the offset distance for each pixel compared to the reference plane. Our measurements show that the systematic depth error depends on the distance to the obstacle and the image region. The error at the image center is in the range of $\pm 6 \mathrm{~mm}$ and increases towards the edges up to $30 \mathrm{~mm}$ for a distance of $2.0 \mathrm{~m}$.

\section{INDOOR EVALUATION}

Further processing of the measurement data (for example for mapping such as in [4]) requires characterization of nonsystematic errors with a suitable statistical model. To take full advantage of the sensor, we are interested in a threedimensional noise distribution of the depth measurements. For this purpose, we empirically derived the parameters of a noise model for the axial (along the sensor camera-axis) and lateral (in directions perpendicular to the camera-axis) errors.

\section{A. Experimental Setup}

Our experimental setup is identical to the setup proposed in [16]. We placed a rotatable planar target in front of the Kinect v2 sensor such that it appears in the center of the image (Fig. 6). Measurements were taken at different $z$-distances and for varying rotation angles $\theta$ (Fig. 6a) of the target around the vertical axis (image y-axis). For a static setup, the axial noise parameter was determined as the standard deviation for each pixel's time-of-flight measurement (Fig. 6p). The lateral noise parameter was obtained from time-of-flight measurements at pixels along the vertical edges of the planar target (Fig. 6.).

Fig. 7 shows the appearance of the target in the color (a), infrared (b), and depth image (c) produced by the Kinect v2.
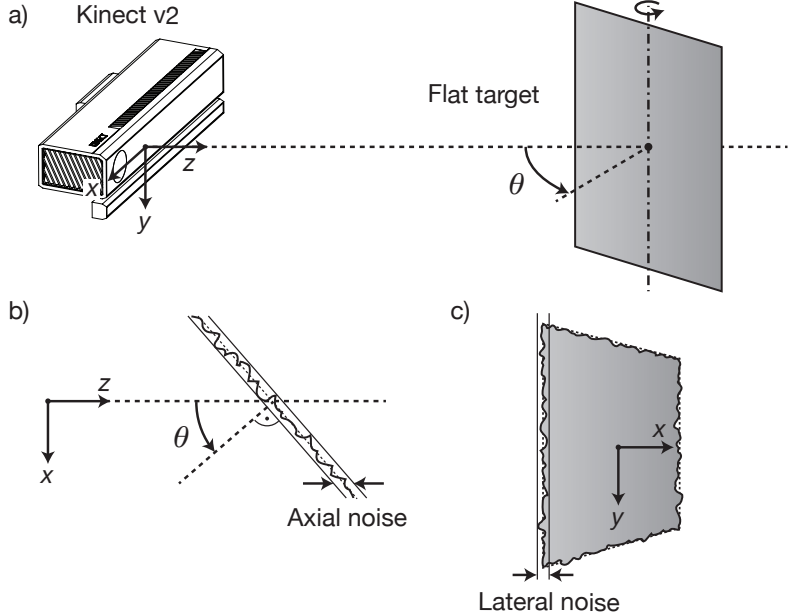

Fig. 6. Experimental setup to evaluate the axial and lateral noise of the Kinect v2 depth sensor. a) The sensor is pointed at a flat target at different distances $z$ and angles $\theta$. b) Top view of the target. The axial noise is estimated from the variation in time of measurements along the $z$-axis. c) Frontal view on the target. Lateral noise is extracted from the variation of the pixels along the edge of the target in the depth image plane. Illustration based on [16].

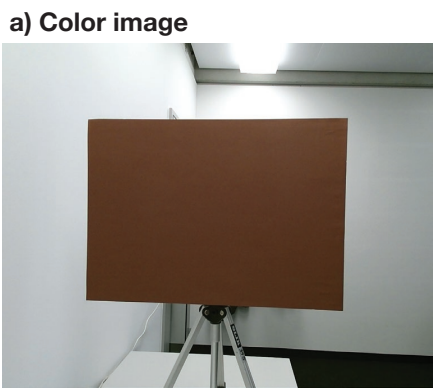

c) Depth image

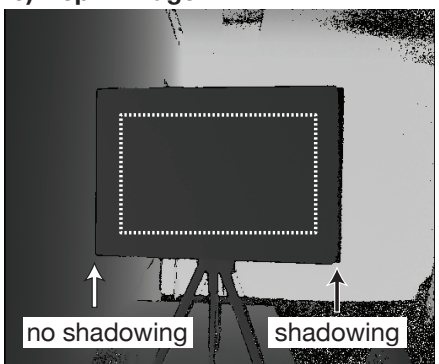

b) Infrared image

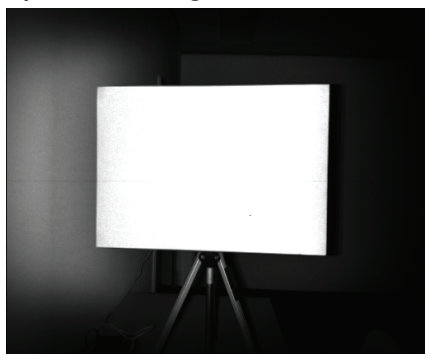

d) Extraction of lateral noise

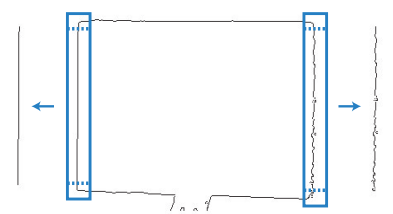

no shadowing

shadowing
Fig. 7. Example data from the Kinect v2 with view on the target from the color camera (a), infrared camera (b) and the depth map (c). Axial noise is extracted from a region inside the target (c) and lateral noise from the cropped left/right borders of the target (d). Different noise magnitudes can be observed for the left and right edge of the target $(c, d)$ due to shadowing of the infrared emitter (left: no shadowing, less noise / right: shadowing, more noise).

We automated the process of extracting the noise parameters by applying a sequence of filters (thresholding, erosion, and canny edge detection) to find corners of the planar target. For the axial noise, we chose an inscribed rectangle with defined margins as interest region to assure that the measurements were not further corrupted by edge effects (see Fig. 7k). For the lateral noise, the edge pixels were extracted by setting regions of interest near to edges of the target and then cropping the upper and lower parts (see Fig. $7 \mathrm{~d}$ ). 


\section{B. Noise Modeling}

The target was placed in front of the camera at varying distances between $0.7 \mathrm{~m}$ and $3.1 \mathrm{~m}$ using $0.3 \mathrm{~m}$ increments. For each target distance, the angle of incidence was varied between $0^{\circ}$ and $75^{\circ}$ with a step size of $15^{\circ}$. To ensure a similar coverage of the target in the resulting fields of view, different target sizes were used depending on the target distance: A2 $(420 \mathrm{~mm} \times 594 \mathrm{~mm})$ for distances between $2.2 \mathrm{~m}$ and $3.1 \mathrm{~m}$, A3 $(297 \mathrm{~mm} \times 420 \mathrm{~mm})$ for distances between $1.9 \mathrm{~m}$ and $1 \mathrm{~m}$, and A4 $(210 \mathrm{~mm} \times 297 \mathrm{~mm})$ for a distance of $0.7 \mathrm{~m}$. For each specific target angle and distance, the noise was determined for each pixel from a series of 100 depth images.

The illuminator and the IR camera are located at slightly different positions in the Kinect v2 (see Fig. 2). This can lead to the effect that shadows become visible in the depth image due to occlusion of the infrared light emitter by foreground objects. In Fig. 7k, the left edge of the target shows no shadowing, while at the right edge, shadowing is apparent. We observed that measurements originating from the shadowingaffected edge were considerably more noisy. For this reason, we differentiate our analysis of the lateral noise with respect to the presence of shadowing.

An example of the axial and lateral noise distributions for a target angle $\theta=0^{\circ}$ is given in Fig. 8. As illustrated, the spread of the axial noise can be approximated well by means of a normal distribution. Although the lateral noise is only partially characterized by a normal distribution, we still provide its empirical standard deviation as a measure, as filtering/fusion frameworks typically assume Gaussian noise distribution. Fig. 9 illustrates the standard deviations of axial and lateral errors over a range of $z$-distances and target rotation angles $\theta$. For the axial noise, the standard deviation $\sigma_{z}$ increases quadratically with the $z$-distance and is $\sigma_{z}<4 \mathrm{~mm}$ for angles $0^{\circ}<\theta<45^{\circ}$, but it increases quickly for angles $\theta>45^{\circ}$. We approximate the standard deviation of the axial noise as a quadratic function of $z[\mathrm{~m}]$ as

$$
\sigma_{z}(z, \theta)[\mathrm{mm}]=1.5-0.5 z+0.3 z^{2}+0.1 z^{\frac{3}{2}} \frac{\theta^{2}}{\left(\frac{\pi}{2}-\theta\right)^{2}},
$$

with a Root-Mean-Squared (RMS) error of the regression of $0.002 \mathrm{~mm}$. Similarly to the model made in [16], we added a hyperbolic term depending on $\theta$ [rad] to account for the growing noise as $\theta$ approaches $90^{\circ}$, and manually determined the factor $z^{\frac{3}{2}}$. As shown in Fig. 9, no clear tendency for the lateral noise $\sigma_{L}$ can be observed. Therefore, we suggest to use a conservative estimate of the lateral noise over the entire range of $z$ and $\theta$ for further applications. Based on the $90^{\text {th }}$ percentile of the standard deviations, conservative values of the standard deviation of the lateral noise are

$$
\begin{aligned}
& \sigma_{L}=1.6 \mathrm{~mm} \text { for edges without shadowing, } \\
& \sigma_{L}=3.1 \mathrm{~mm} \text { for edges with shadowing. }
\end{aligned}
$$

\section{Short-Range Evaluation}

At short distances, the active illumination may cause the IR sensor's cells to over-saturate. We examined the short-range measurement characteristics of the Kinect v2 with an infrared bright and dark target surface as shown in Fig. 10. The obtained depth measurements are categorized in three groups:
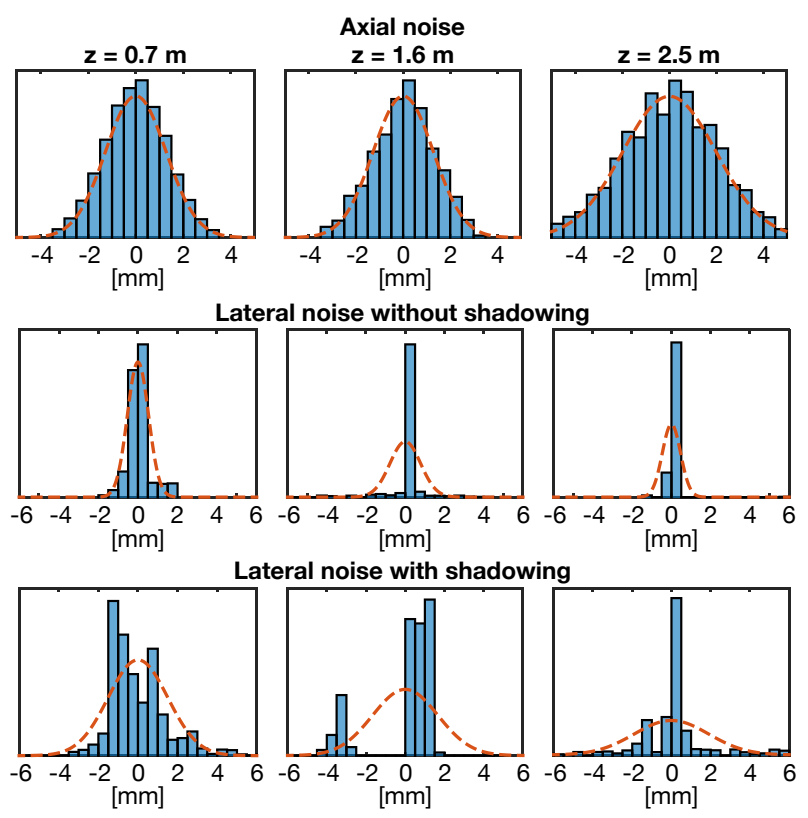

Fig. 8. Histograms illustrating the axial noise (top row), lateral noise without shadowing (middle row), and lateral noise with shadowing (bottom row) for three $z$-distances at target angle $\theta=0^{\circ}$. Approximately $10^{\prime} 000$ depth measurements were taken into account. The fitted normal distributions (using empirical standard deviations) are plotted as dashed line.
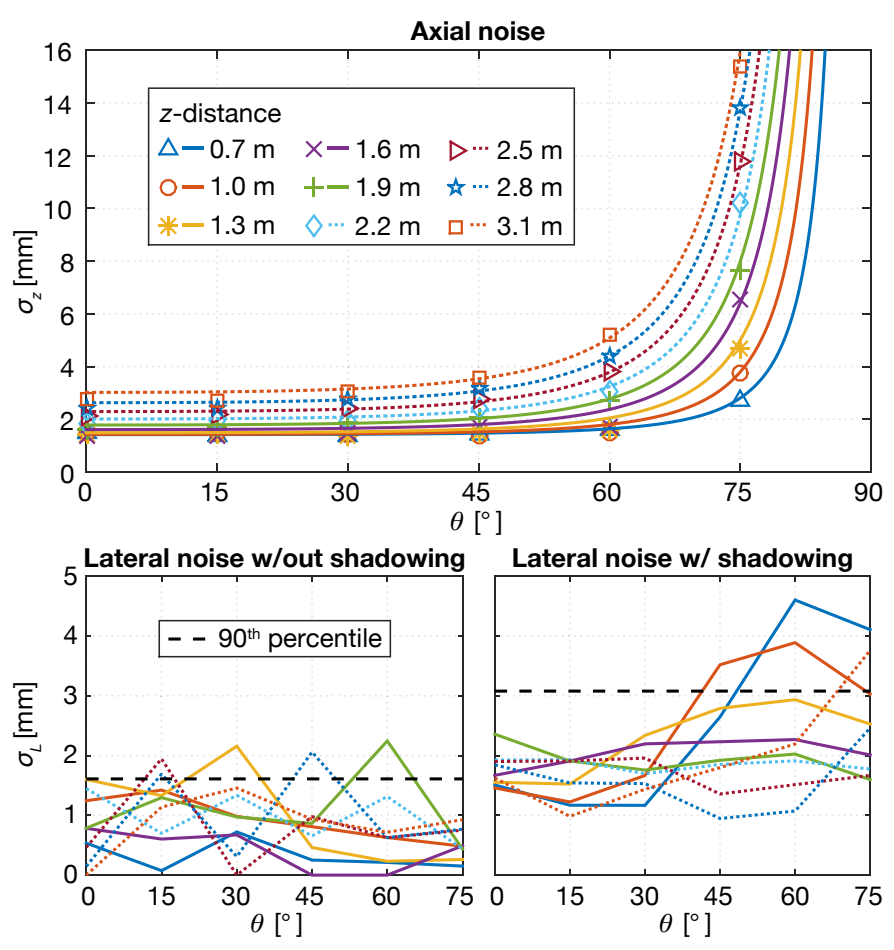

Fig. 9. Measurements and fitted models for axial and lateral noise. The axial noise $\sigma_{z}$ increases quadratically with the measurement distance $z$ and approaches infinity for angles towards $\theta=90^{\circ}$. As no clear tendency arises for the lateral noise $\sigma_{L}$, the $90^{\text {th }}$ percentile is proposed as conservative value for further applications. 


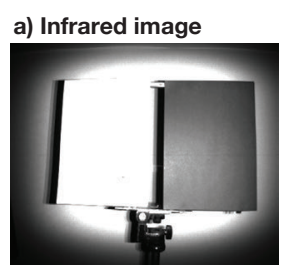

Data categorization
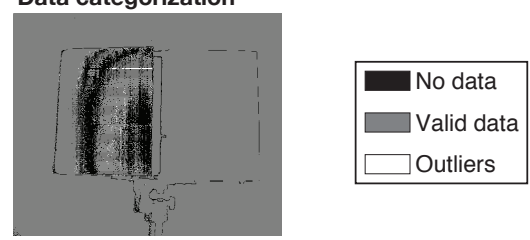

b) Bright surface
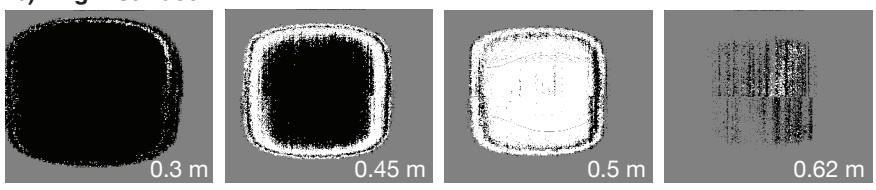

c) Dark surface
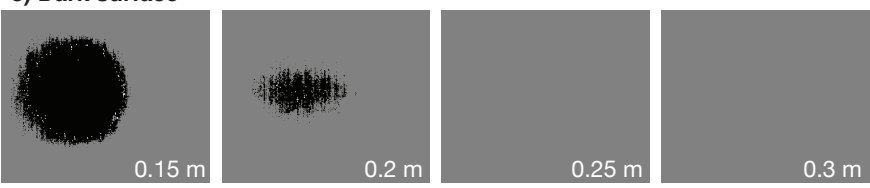

Fig. 10. The minimal distance is examined with infrared bright and dark samples (a). For bright surfaces (b), over-saturation of the IR camera by the active infrared illumination can be observed, leading to invalid measurements. The lower reflectiveness of dark surfaces (c) allows for valid measurements at considerably shorter distances.

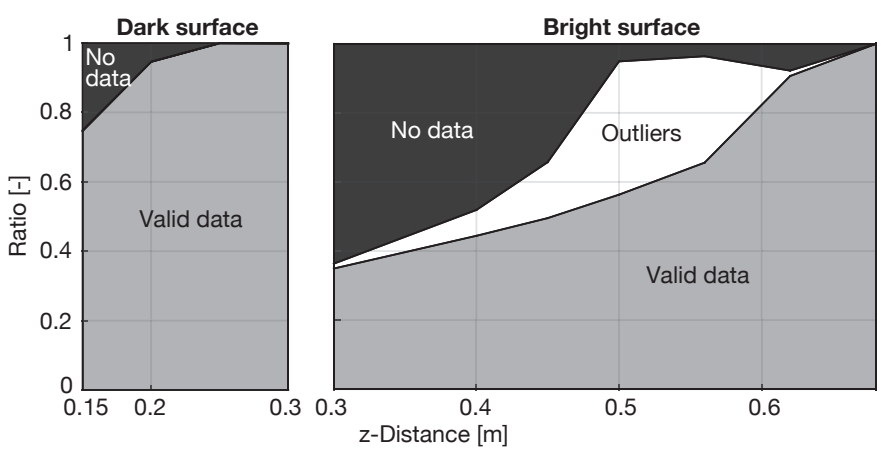

Fig. 11. The ratio between valid data, no data, and outliers, plotted for infrared bright and dark surfaces at short distances. Observing dark surfaces (left), the Kinect v2 sensor is able to reliably measure distances for $z>$ $0.25 \mathrm{~m}$. In the presence of bright surfaces (right), measurements are corrupted for distances up to $0.7 \mathrm{~m}$.

valid data, no data (detected by the sensor), and outliers (determined during post-processing). As shown in Fig. 11. the surface reflectivity has a strong influence on the shortrange measurement performance. With a bright surface, the Kinect v2 reports considerable amounts of missing data and outliers for distances $z<0.7 \mathrm{~m}$. The sensor struggles to detect over-saturation at times which can be observed as an outlier transient between valid and no data. Measurements on a dark surface at short distances are less error-prone, and the Kinect v2 works reliably down to distances of $z=0.25 \mathrm{~m}$.

\section{OUtDOOR EVAluation}

External high intensity infrared light makes it difficult for ToF sensors to differentiate the active infrared signal from external light. For outdoor navigation, we are interested in the depth measurement performance in overcast and direct sunlight situations.

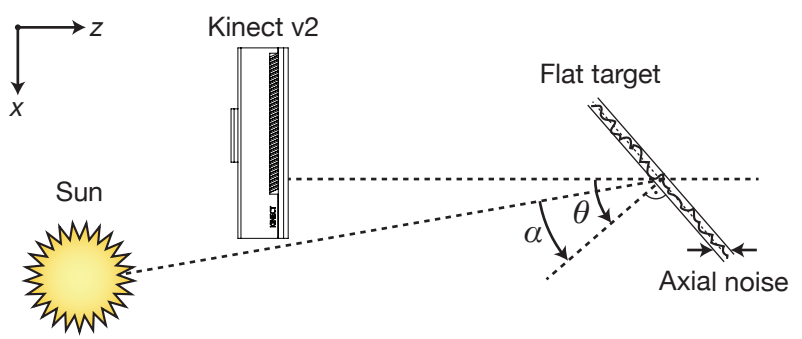

Fig. 12. Top view of the experimental setup for direct sunlight measurements. Additionally to the $z$-distance and target angle $\theta$, we specify the sun incidence angle $\alpha$.

\section{A. Experimental Setup}

We studied the Kinect v2 depth measurement performance in presence of sunlight using the same approach as for our indoor experiments. Assuming an ideal diffusely reflecting surface of the target, we can model the influence of sunlight by describing the angle of incidence with $\alpha$ as depicted in Fig. 12. In order to simplify the measurements, we setup the experiments such that the sun was directly behind the Kinect v2 sensor, i.e., $\alpha=\theta$. The intensity of the environmental light on the target was measured with a lux meter and ranged 1-12 kLux for the overcast and 85-95 kLux for the direct sunlight condition.

\section{B. Noise Characterization}

For the overcast situation, we are able to capture the main characteristics of the noise amplitude by adapting the coefficients of the indoor model (1):

$$
\sigma_{z}(z, \theta)[\mathrm{mm}]=2.5-0.7 z+0.9 z^{2}+0.5 z^{\frac{3}{2}} \frac{\theta^{2}}{\left(\frac{\pi}{2}-\theta\right)^{2}},
$$

with a resulting RMS regression error of $0.55 \mathrm{~mm}$ (compared to $0.002 \mathrm{~mm}$ for indoors). We found that ranges between $1.0-2.8 \mathrm{~m}$ could reliably be measured, whereas measurements outside this range were considered as unreliable and therefore omitted from the noise model fitting. For the lateral noise, we again found the $90^{\text {th }}$ percentile of the standard deviation as a fixed value with

$$
\sigma_{L}=6.8 \mathrm{~mm}
$$

without the consideration of shadowing effects. Our measurements indicated that, by exposing the Kinect v2 to overcast light, the axial and lateral noise in the depth measurements must be expected to increase by factor 2-3.

During direct sunlight exposure, a strong influence of the sensor-to-target distance and the sunlight incidence angle $\alpha$ was observed. Fig. 13a illustrates the difference between overcast and direct sunlight environments. While $100 \%$ valid measurements could be acquired at a distance of $z=1.6 \mathrm{~m}$ in overcast weather, only little valid data was captured using the same setup in direct sunlight. The rapid degradation of the measurement data for a target angle of $\theta=15^{\circ}$ and increasing target distances is shown in Fig. 13 p. An analysis for the influence of the measurement distance and sunlight incidence angle on the ratio of valid data, outliers, and no data is given in Fig. 14. For distances $z<1.0 \mathrm{~m}$, reliable measurements could be acquired even under perpendicular sunlight illumination of the target plane. The amount of valid data dropped for increased $z$-distances until essentially no data could be gathered for distances $z>1.9 \mathrm{~m}$. We also observed that the sunlight incidence 
a)
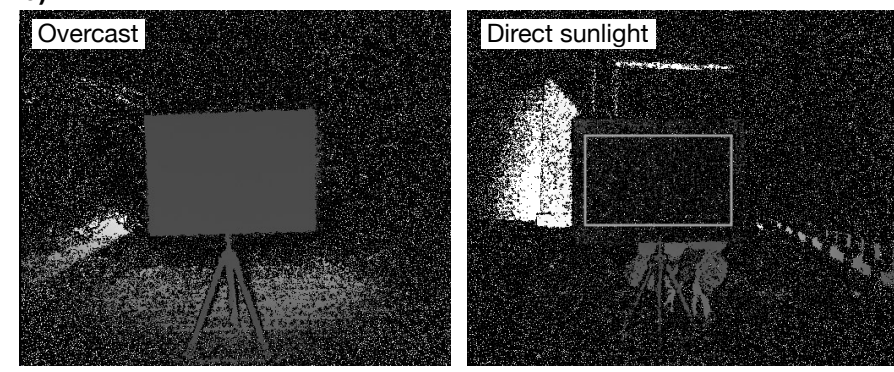

b) Direct sunlight
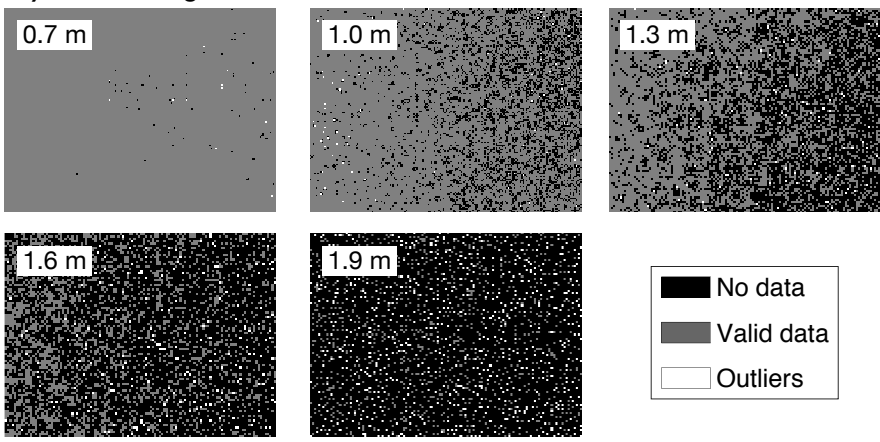

Fig. 13. The influence of direct sunlight on the depth measurement data quality is illustrated with sample images. a) The comparison between overcast and direct sunlight reveals a significant reduction of data quality in sunlight for the same target distance $z=1.6 \mathrm{~m}$. b) Sample images of the target plane at angle $\theta=\alpha=15^{\circ}$ indicate the rapid increase in the amount of invalid measurements for increasing distances.
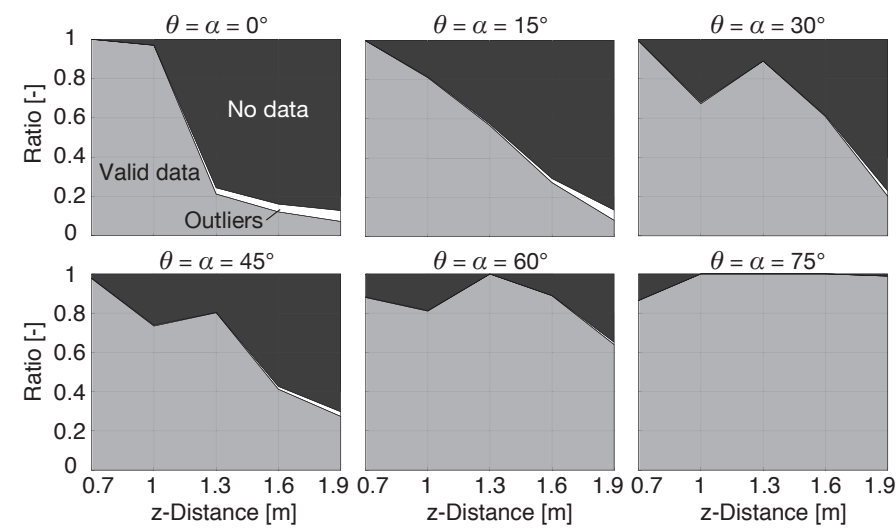

Fig. 14. The ratio between valid data, no data, and outliers is illustrated for the direct sunlight scene over a range of distances $z$ and angles $\theta=\alpha$. Higher $z$-distances and more direct sunlight on the target (small $\alpha$ ) decrease the reliability of the Kinect v2 to take valid measurements.

angle considerably influences the measurement performance. In particular, we found that more direct incidence (maximal at $\alpha=0^{\circ}$ ) caused stronger degradation of the data than less direct incidence (such as $\alpha=75^{\circ}$ ). This behavior is also reflected in the noise amplitude characteristics. Fig. 15 illustrates the axial standard deviation $\sigma_{z}$ as a function of the target angle $\alpha=\theta$. In general, the measurement noise for direct sunlight environments is roughly an order of magnitude higher than for indoor scenes. Since we have determined the sensor's noise characteristics without environmental light influence (Fig. 9), we may argue that the increase in noise for smaller angles $\theta=\alpha$ is due to the presence of sunlight. As suggested by Lambert's cosine law, the intensity observed by the target (assumed to be ideally diffusely reflecting) is proportional to

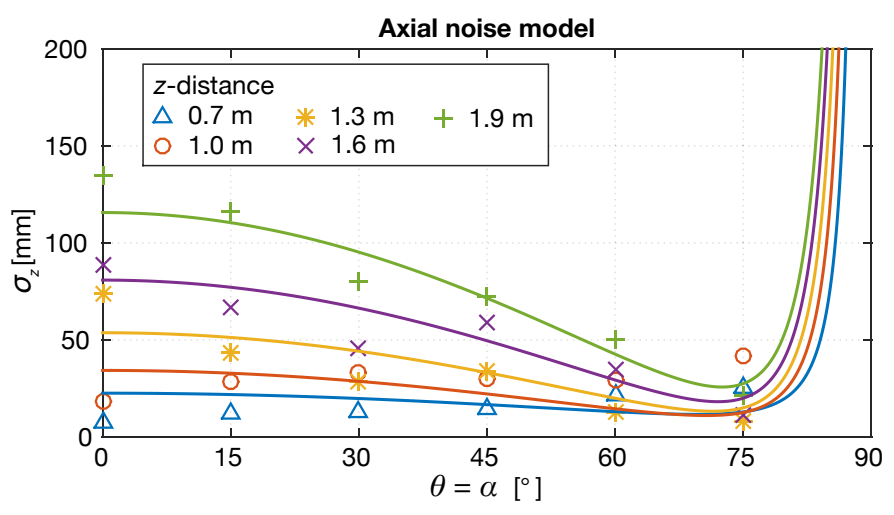

Fig. 15. Plot of the empirically determined axial noise standard deviation $\sigma_{z}$ for direct sunlight. To simplify the experiments, measurements were taken with the sun located directly behind the sensor, such that $\alpha=\theta$. The noise magnitude increases with the measurement distance $z$ and for lower incidence angles $\alpha=\theta$. The plotted lines correspond to an extended model fit which takes the sunlight angle $\alpha$ into account.

the cosine of the incidence angle $\alpha$. We extend our existing model accordingly by introducing the dependency on $\cos \alpha$ as

$$
\begin{aligned}
& \sigma_{z}(z, \theta, \alpha)[\mathrm{mm}] \\
& =28-38 z+2.0 z^{2}+0.3 z^{\frac{3}{2}} \frac{\theta^{2}}{\left(\frac{\pi}{2}-\theta\right)^{2}}+42 z^{2} \cos \alpha,
\end{aligned}
$$

where the factor $z^{2}$ was determined manually. The resulting model describes the noise characteristics with an RMS regression error of $4.4 \mathrm{~mm}$.

\section{CONCLUSION}

In this work, an evaluation of the second version Kinect depth sensor for mobile robot navigation was presented. We have given an analysis of the systematic errors and the short-range measurement capability. For further processing of the data, empirical models approximating the axial and lateral noise amplitudes were proposed. In indoor and overcast environments, the model represents a function of the measurement distance and surface angle. To account for the effects of direct sunlight exposure, this model was extended with a term capturing the incidence angle of the sunlight. Table [II summarizes a quantitative comparison of the noise characteristics for the original Kinect and the Kinect v2 in indoors, overcast and direct sunlight situations. The values are given for two configurations, [A: $z=1.0 \mathrm{~m}, \theta=45^{\circ}$ ] and [B: $z=2.8 \mathrm{~m}, \theta=10^{\circ}$ ], which both correspond to representative configurations for a Kinect sensor mounted on a mobile robot. The Kinect v2 shows significantly lower axial noise magnitudes than the original Kinect, especially at larger distances (configuration B). For the lateral noise, the two versions of the sensor display similar performances. The Kinect v2 can be used outdoors in overcast situations as valid measurements can be acquired for ranges up to $\sim 2.8 \mathrm{~m}$. However, a 2-3 fold amplification of the noise must be expected in comparison to indoor scenes. In direct sunlight, the data quality of the Kinect v2 strongly depends on the distance to the target and the incidence angle of the sunlight. Whereas a reasonable amount of data was captured in configuration $\mathrm{A}$, no data could be acquired under configuration B. Although valid measurements may be obtained with the Kinect v2 in direct sunlight for distances up to $\sim 1.9 \mathrm{~m}$, an 
TABLE II. ORIGINAL KINECT AND KINECT v2 COMPARISON

\begin{tabular}{llcccc}
\hline & $z=$, & Original Kinect $^{\mathrm{a}}$ & \multicolumn{3}{c}{ Kinect v2 } \\
& $\theta=\alpha=$ & Indoors & Indoors & Overcast & Sunlight \\
\hline \multirow{2}{*}{ Axial noise } & A: $1.0 \mathrm{~m}, 45^{\circ}$ & $2.0 \mathrm{~mm}$ & $1.4 \mathrm{~mm}$ & $3.2 \mathrm{~mm}$ & $22 \mathrm{~mm}$ \\
& B: $2.8 \mathrm{~m}, 10^{\circ}$ & $12 \mathrm{~mm}$ & $2.5 \mathrm{~mm}$ & $7.6 \mathrm{~mm}$ & - \\
\hline \multirow{2}{*}{ Lateral noise } & A: $1.0 \mathrm{~m}, 45^{\circ}$ & $1.4 \mathrm{~mm}$ & $1.6^{\mathrm{b}} / 3.1^{\mathrm{c}} \mathrm{mm}$ & $6.8 \mathrm{~mm}$ & - \\
& B: $2.8 \mathrm{~m}, 10^{\circ}$ & $3.9 \mathrm{~mm}$ & $1.6^{\mathrm{b}} / 3.1^{\mathrm{c}} \mathrm{mm}$ & $6.8 \mathrm{~mm}$ & - \\
\hline \multirow{2}{*}{ Valid data } & A: $1.0 \mathrm{~m}, 45^{\circ}$ & - & $100 \%$ & $100 \%$ & $73 \%$ \\
\multicolumn{1}{l}{ B: $2.8 \mathrm{~m}, 10^{\circ}$} & - & $100 \%$ & $100 \%$ & $0 \%$ \\
\multicolumn{2}{c}{ a Data for the near-mode taken from Nguyen et al. [16] } \\
bithout shadowing $/{ }^{\mathrm{c}}$ with shadowing
\end{tabular}

increase in noise by approximately an order of magnitude should be expected.

Based on our experiments and experiences with the Kinect v2, we conclude that the system has several beneficial properties for mobile robot navigation. Thanks to the ToF technology, robust depth measurements can be acquired even in textureless or poorly lit scenes. A vast amount of data $(6.5 \mathrm{mio}$. points/s) is captured by the Kinect v2 due to its high resolution compared to the original Kinect and other ToF sensors. This allows mapping algorithms to model the environment in fine detail and to detect thin obstacles. The Kinect v2 provides a big improvement over the original Kinect for outdoor/sunlight situations. Whereas the original version is not suited to outdoor usage, the Kinect v2 can measure depth at ranges below $\sim 2 \mathrm{~m}$. Additionally, the Kinect v2 includes a high-quality color camera and an IR camera with active illumination, providing an image of the surroundings even in dark environments.

However, certain limitations should also be considered when implementing the Kinect v2 on a mobile robot. The second version of the Kinect is only suited for robots where the device's bigger size, higher mass, and increased power consumption can be tolerated. The minimal measurement distance may cause objects close the robot to not be recognizable by the sensor. Depending on the application, the field of view of the Kinect v2 can be limiting when compared, for example, to a rotating laser scanner. Furthermore, noise and outlier characteristics, especially in direct sunlight, lead to corruptions of the depth measurements. Hence, a suitable postprocessing framework is required to omit artifacts from faulty measurements in the succeeding processing steps.

Our investigations of the Kinect v2 provides first insights into the prospective value of the sensor to mobile robot applications. However, certain aspects need to be explored in future work. For instance, the question of how to switch or interpolate between the environment dependent models (indoors, overcast, and direct sunlight) needs to be addressed. We image this could be solved with an additional sensor to measure the light intensity of the environment or estimating the external light online from the measurement noise. Also, more advanced calibration procedures should be derived to further minimize the systematic errors. Although a fan regulates the heating of the sensor, it seems worth studying the influence of the ambient temperature for a range of thermal conditions associated with outdoor usage. Particularly for fast moving robots or nonstationary environments, the effects of motion blur might deteriorate the depth measurements. It would further be interesting to experiment with hardware/software modifications to reduce the minimal measurement distance. An experimental near-field firmware has been demonstrated by the manufacturer [17] to reduce the measurement range to $0.1-1.0 \mathrm{~m}$, but has not yet been released to the best of our knowledge.

\section{ACKNOWLEDGMENT}

The authors would like to thank Joshua Blake, Florian Echtler, and Christian Kerl for their contributions to libfreenect2 [13]. We are much obliged to Thiemo Wiedemeyer from the University of Bremen for his work on IAI Kinect2 [14]. Many thanks to Andreas Lauber for the CAD modeling of the Kinect v2.

This work was supported in part by the Swiss National Science Foundation (SNF) through project 200021_149427 / 1 and the National Centre of Competence in Research Robotics.

\section{REFERENCES}

[1] R. A. El-Iaithy, J. Huang, and M. Yeh, "Study on the Use of Microsoft Kinect for Robotics Applications," in Position Location and Navigation Symposium (PLANS). IEEE/ION, 2012.

[2] Microsoft, "Kinect for Windows features," http://www.microsoft.com/ en-us/kinectforwindows/meetkinect/features.aspx January, 2015.

[3] M. Hutter, C. Gehring, M. Bloesch, M. A. Hoepflinger, C. D. Remy, and R. Siegwart, "StarlETH: A compliant quadrupedal robot for fast, efficient, and versatile locomotion," in International Conference on Climbing and Walking Robots (CLAWAR), 2012.

[4] P. Fankhauser, M. Bloesch, C. Gehring, M. Hutter, and R. Siegwart, "Robot-Centric Elevation Mapping with Uncertainty Estimates," in International Conference on Climbing and Walking Robots (CLAWAR), Poznan, Poland, 2014.

[5] S. Zennaro, "Evaluation of Microsoft Kinect 360 and Microsoft Kinect One for robotics and computer vision applications," Master's thesis, University of Padova, Italy, 2014.

[6] T. Breuer, C. Bodensteiner, and M. Arens, "Low-cost Commodity Depth Sensor Comparison and Accuracy Analysis," in SPIE Security+ Defence, vol. 9250. International Society for Optics and Photonics, 2014.

[7] A. M. Pinto, P. Costa, A. P. Moreira, L. F. Rocha, G. Veiga, and E. Moreira, "Evaluation of Depth Sensors for Robotic Applications," in IEEE International Conference on Autonomous Robot Systems and Competitions, 2015.

[8] T. Butkiewicz, "Low-cost Coastal Mapping using Kinect v2 Time-ofFlight Cameras," in Oceans - St. John's. IEEE, 2014.

[9] E. Lachat, H. Macher, M.-A. Mittet, T. Landes, and P. Grussenmeyer, "First Experiences With Kinect V2 Sensor for Close Range 3D Modelling," in International Archives of the Photogrammetry, Remote Sensing and Spatial Information Sciences (ISPRS), 2015.

[10] S. Foix, G. Alenya, and C. Torras, "Lock-in Time-of-Flight (ToF) Cameras: A Survey," IEEE Sensors Journal, vol. 11, no. 3, pp. 1-11, 2011.

[11] J. Sell and P. O'Connor, "The Xbox One System on a Chip and Kinect Sensor," IEEE Micro, vol. 34, no. 2, pp. 44-53, 2014.

[12] iFixit, "Xbox One Kinect Teardown," https://www.ifixit.com/Teardown/ Xbox+One+Kinect+Teardown/19725 January, 2015.

[13] J. Blake, F. Echtler, and C. Kerl, "libfreenect2: Open source drivers for the Kinect for Windows v2 device," https://github.com/OpenKinect/ libfreenect2. January, 2015.

[14] T. Wiedemeyer, "IAI Kinect2: Tools for using the Kinect One (Kinect v2) in ROS," https://github.com/code-iai/iai_kinect2. January, 2015.

[15] D. Lefloch, R. Nair, F. Lenzen, H. Schäfer, L. Streeter, M. J. Cree, R. Koch, and A. Kolb, "Technical foundation and calibration methods for time-of-flight cameras," Time-of-Flight and Depth Imaging. Sensors, Algorithms, and Applications, pp. 3-24, 2013.

[16] C. V. Nguyen, S. Izadi, and D. Lovell, "Modeling Kinect Sensor Noise for Improved 3D Reconstruction and Tracking," in International Conference on $3 D$ Imaging, Modeling, Processing, Visualization and Transmission, 2012.

[17] Microsoft, "Hackers put Kinect for Windows v2 through its paces," http://blogs.msdn.com/b/kinectforwindows/archive/2014/07/ 02/hackers-put-kinect-for-windows-v2-through-its-paces.aspx January, 2015. 